\title{
Editorial
}

\section{Production and Application of Petroleum Oil and Its Alternatives on Internal Combustion Engines}

\author{
Haifeng Liu, ${ }^{1}$ Zheng Chen, ${ }^{2}$ Ming Huo, ${ }^{3}$ and Chao Jin ${ }^{4}$ \\ ${ }^{1}$ State Key Laboratory of Engines, Tianjin University, Tianjin 300072, China \\ ${ }^{2}$ Department of Energy \& Power Engineering, Hunan University, Changsha, Hunan 410082, China \\ ${ }^{3}$ EcoMotors Inc., Allen Park, MI 48101, USA \\ ${ }^{4}$ School of Environmental Science and Engineering, Tianjin University, Tianjin 300072, China
}

Correspondence should be addressed to Haifeng Liu; haifengliu@tju.edu.cn

Received 9 June 2015; Accepted 10 June 2015

Copyright (C) 2015 Haifeng Liu et al. This is an open access article distributed under the Creative Commons Attribution License, which permits unrestricted use, distribution, and reproduction in any medium, provided the original work is properly cited.

Internal combustion engines are widely used in vehicles, ships, construction machinery, and agricultural machinery due to their reliability, durability, and high efficiency. Furthermore, they will be the main powertrains for above powerplant in the next 50 years according to the wide studies. However, the development of engines is faced with some serious challenges in the future, such as the stringent emission regulations, petroleum energy supply, and greenhouse gas emissions. Therefore, we need to develop some advanced combustion technologies to reduce the pollutant emissions. Meanwhile, to prevent global warming, we need to improve the thermal efficiencies of engines and thereby reduce $\mathrm{CO}_{2}$ emissions and save petroleum oil.

Fuel properties have remarkable effects on engine performance and emissions. High-efficiency and low-emission engines must need the cooperation of high-quality fuels. Meantime, the future advanced engines may need the improved fuel properties which are different from the current gasoline or diesel fuel. In addition, the alternative biofuels have recently gained significant political and scientific interests owing to the concerns about climate change, global energy security, and petroleum supply shortage in the foreseeable future.

In this special issue, firstly, N. Li et al. from State Key Laboratory of Oil and Gas Reservoir Geology and Exploitation, Southwest Petroleum University, present the improvement on production of petroleum oil. Secondly, C. Jin and Z.Q. Zheng from Tianjin University and B. Yang et al. from
Shandong University of Technology present the need for engine fuel properties for future advanced combustion modes such as homogeneous charge compression ignition and low temperature combustion. Finally, L. Dong et al. from Civil Aviation University of China and F. Zhang et al. from Hunan University present how to achieve high-efficiency and clean combustion based on cooperated control of fuel, oil, and engines.

Finally, we hope this special issue can give you some help to understand the tight relationship between petroleum and engines and to know the future engine combustion technologies and the needs for future engine fuels and oil.

Haifeng Liu Zheng Chen Ming Huo Chao Jin 

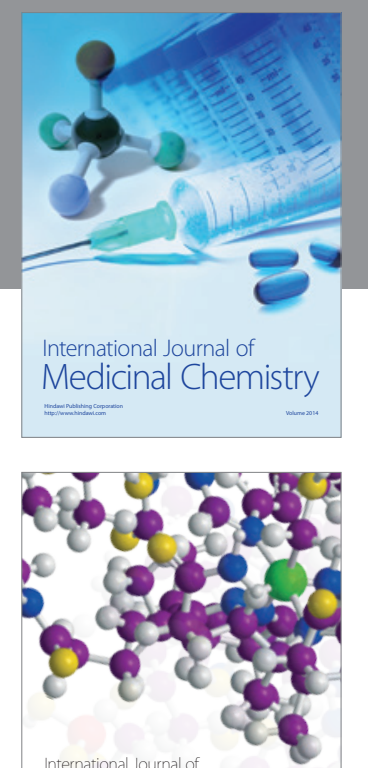

\section{Carbohydrate} Chemistry

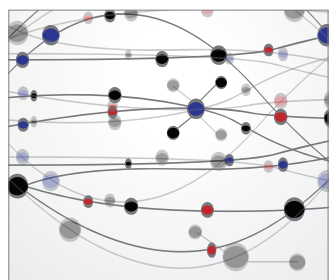

The Scientific World Journal
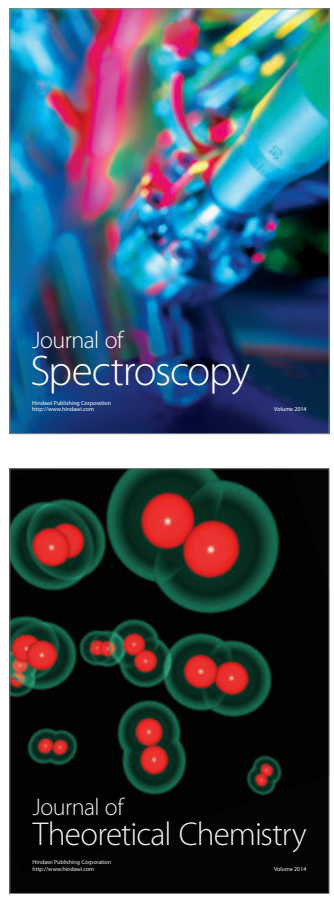
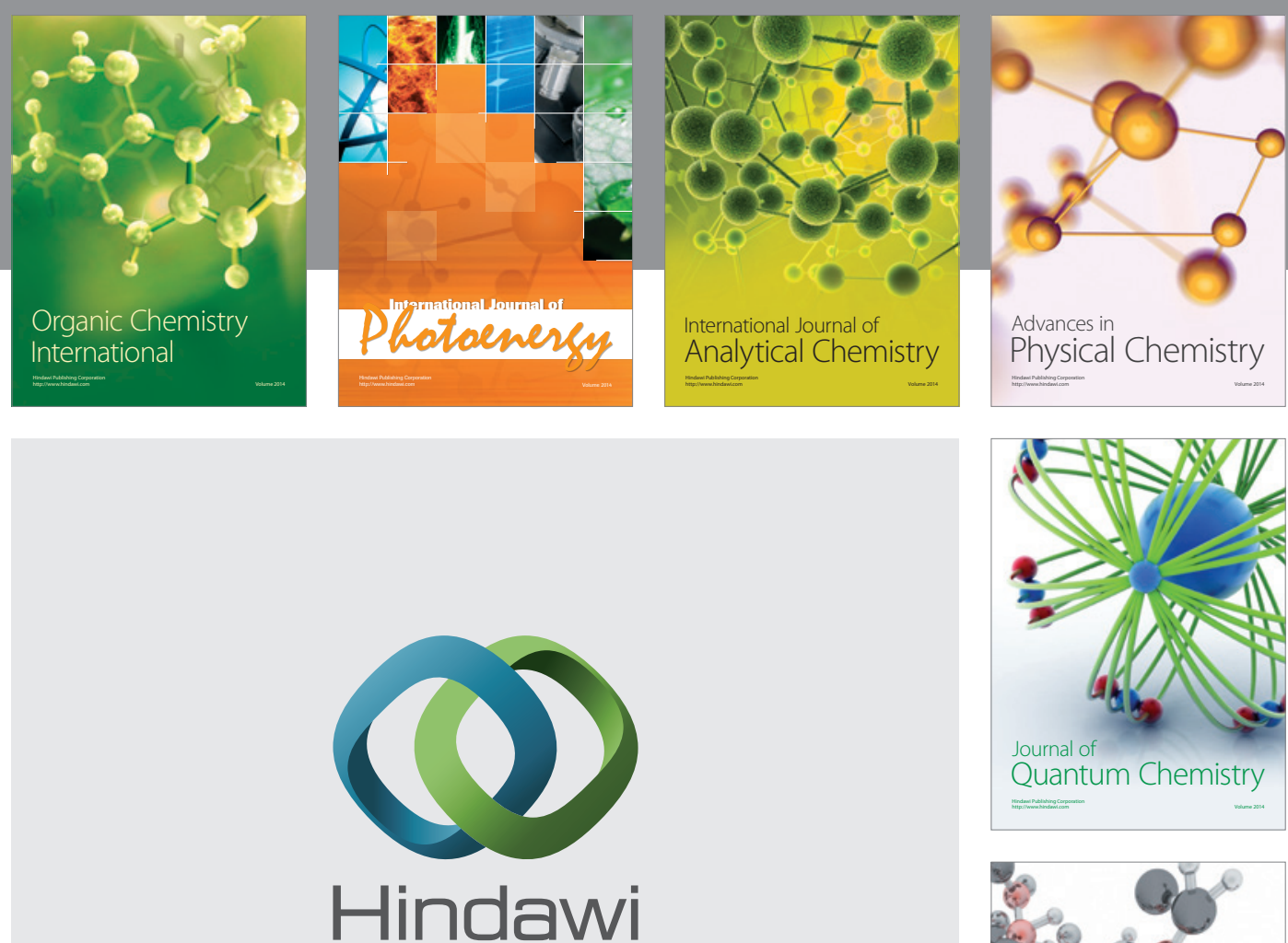

Submit your manuscripts at

http://www.hindawi.com

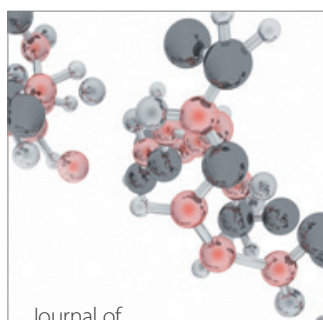

Analytical Methods

in Chemistry

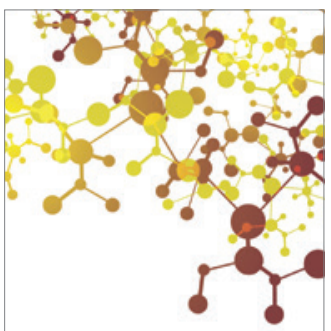

Journal of

Applied Chemistry

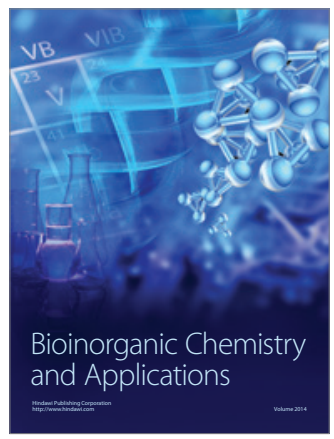

Inorganic Chemistry
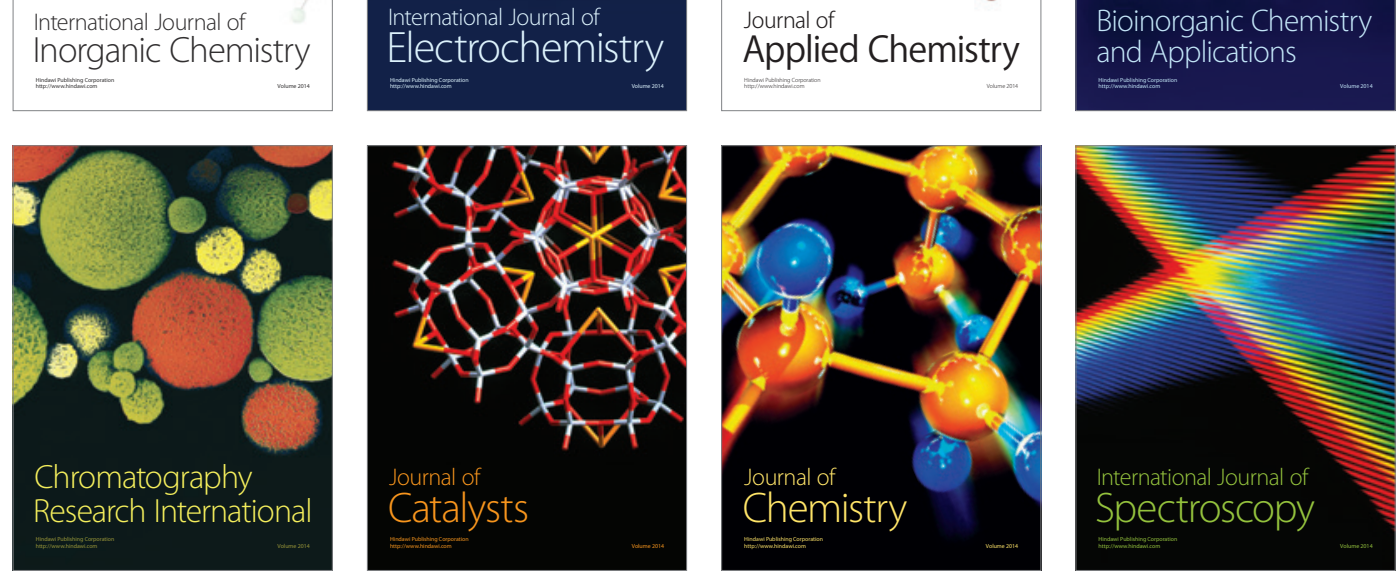